\title{
Large-scale star formation in galaxies
}

\section{The spirals NGC 3377A, NGC 3507 and NGC 4394. Young star groupings in spirals}

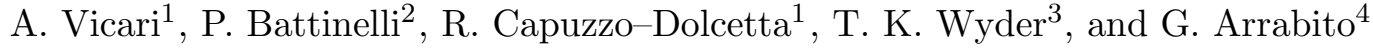 \\ 1 Dipart. di Fisica, Università La Sapienza, P.le A. Moro 5, Roma, Italy \\ 2 Oss. Astronomico di Roma, Viale del Parco Mellini 84, Roma, Italy \\ 3 California Inst. of Technology MC 405-47 1200 E. Cal. Blvd Pasadena, CA 91125, USA \\ 4 Dipart. di Matematica, Università La Sapienza, P.le A. Moro 5, Roma, Italy
}

Received 28 June 2001 / Accepted 23 November 2001

\begin{abstract}
The identification of young star groupings (YSG) in the three spiral galaxies NGC 3377A, NGC 3507, NGC 4394 is obtained by mean of the statistical method described in Paper I. We find 83, 90, 185 YSGs, respectively. An identification map of YSGs, as well as their size distribution, their B-luminosity function and their surface luminosity density radial behaviour are presented and compared. These data, in addition to those in Paper I, constitute a first sample suitable for seeking correlations among properties of galaxies and their YSGs, which we briefly discuss here.
\end{abstract}

Key words. galaxies: spiral - galaxies: general - galaxies: stellar content - stars: formation - methods: statistical

\section{Introduction}

Global star formation in a spiral galaxy is influenced by several factors. Different authors have investigated the dependence of the star formation rate and efficiency on environment. Star formation in interacting galaxies is generally enhanced, while its dependence upon galaxy morphological type is not well assessed (Young et al. 1996 and references therein). Some recent papers have shown the importance of the determination of empirical correlations between large-scale star forming regions and parent galaxy properties in understanding the star formation process. In this framework Elmegreen et al. $(1994,1996)$ and Elmegreen \& Salzer (1999), analyzing different samples of spirals, have found a clear correlation between the size, the total $B$ magnitude of a galaxy and the size of its largest stellar complex. These authors suggest that these scaling laws can be related to the properties of the interstellar medium and of the instability process itself. However, we note that Selman \& Melnick (2000) have recently explained that the correlation between the size of the largest superassociation and the luminosity of the parent galaxy,

Send offprint requests to: A. Vicari, e-mail: alessandrovicari@uniroma1.it found by Elmegreen et al. (1994), is most likely due to a size-of-sample effect.

The problem of the identifications of regions of star formation in distant, unresolved, galaxies is quite difficult, because the identification suffers from several biases, like those introduced by differences in observational data and/or in the identification criteria adopted. Hodge (1986) discussed these difficulties when trying to derive general properties of star forming regions.

To overcome these complications we developed an automatic method for the identification of the star forming regions (Young Star Groupings, or YSGs hereafter) in unresolved galaxies (Adanti et al. 1994). The availability of various sets of colours and fluxes allows every pixel of the galaxy image to be represented as a point in the space of the variables (i.e. of fluxes and colours). Principal Component Analysis (PCA) and Cluster Analysis (CA) result in an artificial image of the galaxy, where pixels are grouped into classes according to their relative distances in the space of the variables. As in Battinelli et al. (2000, hereafter Paper I), we performed our classification using $U, U-B, B-V$ and $B-R$ as variables. A first step to a deeper understanding of the link between the YSG's and parent galaxy's properties is to build a homogeneous and 
"objective" database. Target galaxies were chosen according to the criteria discussed in Paper I.

In this paper we present data and results of YSGs in three spiral galaxies: NGC 3337A, NGC 3507 and NGC 4394. In Sect. 2 observational data acquisition and reduction are described; in Sect. 3 we present and discuss our identifications in each galaxy. Finally, in Sect. 4, we discuss some properties of the sample of YSGs in all the six galaxies we have studied so far.

\section{Observations and data reduction}

All data were obtained with the SPIcam camera at the $3.5 \mathrm{~m}$ telescope of the Apache Point Observatory (New Mexico, USA $)^{1}$, in four different broadbands: $U, B$, $V$ and $R$. SPIcam is a backside illuminated thinned SITe CCD, with a size of $1024 \times 1024$ pixels, with an angular scale of $0.28^{\prime \prime} /$ pixel and a field of view of about $4.8^{\prime} \times 4.8^{\prime}$.

Three exposures per filter were taken for each galaxy. The CCDPROC routine in $\mathrm{IRAF}^{2}$ was used to subtract the bias, as measured in the overscan region of each image, as well as to divide by the appropriate flat field for that night and filter. The spatial offsets among the images of a particular galaxy were obtained using the positions of stars within each field. The images were shifted to a common reference frame using the IMALIGN routine in IRAF. Finally, the IRAF task IMCOMBINE was used to average the three exposures in each filter while at the same time rejecting cosmic rays.

NGC 3377A was observed on the night of 1998 November 21, a photometric night, while the NGC 3507 and NGC 4394 images were taken on 1998 December 21 and on 17-18 January 1999 respectively. These last three nights were not photometric, so we obtained shallower exposures of NGC 3507 and of NGC 4394 on a clear night, 16 April 1999, which we used to calibrate these images. The ratio in each filter between the averaged images of the non-photometric nights and the shallower images taken on the last photometric night was determined using stars within the field, if present, or using the radial profile of the galaxy itself. Total exposure times are always $1200 \mathrm{~s}$ in the $U$ images and $600 \mathrm{~s}$ in the $B, V$ and $R$ bands, with the exception of the $U$ image of NGC 4394, whose exposure time is $2000 \mathrm{~s}$. The seeing of each image is reported in Table 1.

As mentioned in Paper I, the flat fields (one for each night and for each band) may be contaminated by scattered light from the lack of baffling of the $3.5 \mathrm{~m}$ APO

${ }^{1}$ APO is privately owned and operated by the Astrophysical Research Consortium (ARC), consisting of the University of Chicago, the Institute for Advanced Study, Johns Hopkins University, New Mexico State University, Princeton University, the University of Washington and Washington State University.

${ }^{2}$ IRAF is a free software distributed by National Optical Astronomy Observatories, which are operated by the Association of Universities for Research in Astronomy Inc., under contract to the National Science Foundation.
Table 1. Observational log.

\begin{tabular}{|l|c|c|c|c|}
\hline \hline & Filter & $\begin{array}{c}\text { Date } \\
(\mathrm{d} / \mathrm{m} / \mathrm{y})\end{array}$ & $\begin{array}{c}\text { Exp. time } \\
(\mathrm{s})\end{array}$ & $\begin{array}{c}F W H M \\
(\mathrm{arcsec})\end{array}$ \\
\hline NGC 3377A & $U$ & $21 / 11 / 98$ & $3 \times 400$ & 1.18 \\
& $B$ & $21 / 11 / 98$ & $3 \times 200$ & 1.18 \\
& $V$ & $21 / 11 / 98$ & $3 \times 200$ & 1.40 \\
& $R$ & $21 / 11 / 98$ & $3 \times 200$ & 1.18 \\
\hline NGC 3507 & $U$ & $21 / 12 / 98$ & $3 \times 400$ & 1.04 \\
& $B$ & $21 / 12 / 98$ & $3 \times 200$ & 1.12 \\
& $V$ & $21 / 12 / 98$ & $3 \times 200$ & 0.92 \\
& $R$ & $21 / 12 / 98$ & $3 \times 200$ & 0.84 \\
\hline NGC 4394 & $U$ & $17 / 01 / 99$ & $2 \times 800,1 \times 400$ & 1.12 \\
& $B$ & $18 / 01 / 99$ & $3 \times 200$ & 1.06 \\
& $V$ & $18 / 01 / 99$ & $3 \times 200$ & 0.90 \\
& $R$ & $18 / 01 / 99$ & $3 \times 200$ & 0.92 \\
\hline \hline
\end{tabular}

telescope, leading to an artificial spatial gradient in the sky background. This is more evident in the $B$ and $R$ band images than in the $U$ and $B$ ones. The one exception is NGC 4394 where some of the gradient in the background is due to the presence of the galaxy M 85, just off the field of view.

Calibration was performed using observations of several standard stars from Landolt (1992). An aperture magnitude for each observation of each standard star was obtained with an aperture radius of $7^{\prime \prime}$, chosen to match the aperture used by Landolt. Standards included 3 stars in the field of PG 1323-086 and 5 stars in the field of PG $1633+099$, with color index in the range from -0.9 to 1.1 for $U-B$, from -0.2 to 1.1 for $B-V$ and from -0.1 to 0.6 for $V-R$. The standards were mostly observed at low airmass, less than 1.2 , with the entire range being from 1.1 to 2.2 .

\section{Results}

\subsection{NGC 3377 A}

NGC 3377A is an almost face-on dwarf spiral galaxy, located about $7^{\prime}$ north-west from the E6 giant elliptical NGC 3377, in the Leo I (M96) group. The radial velocity difference of $120 \mathrm{~km} \mathrm{~s}^{-1}$ (de Vaucouleurs et al. 1991, hereafter RC3) between these two galaxies suggests that NGC 3377A may be a companion of the giant elliptical. According to Tonry et al. (1997), NGC 3377A is about $10.7 \mathrm{Mpc}$ distant, implying a linear separation from NGC 3377 of only $20 \mathrm{kpc}$. Sandage et al. (1991) and Knezek et al. (1999) showed that this very low surface brightness galaxy has various peculiarities. In particular, in spite of its high $M_{\mathrm{HII}} / L_{\mathrm{B}}$ ratio $(\sim 0.30$ in solar units, which is a typical value for a Sc type spiral), it has a very low star formation rate, about $0.003 M_{\odot} / \mathrm{yr}$, so that star formation can continue for more than a Hubble time (Knezek et al. 1999). 


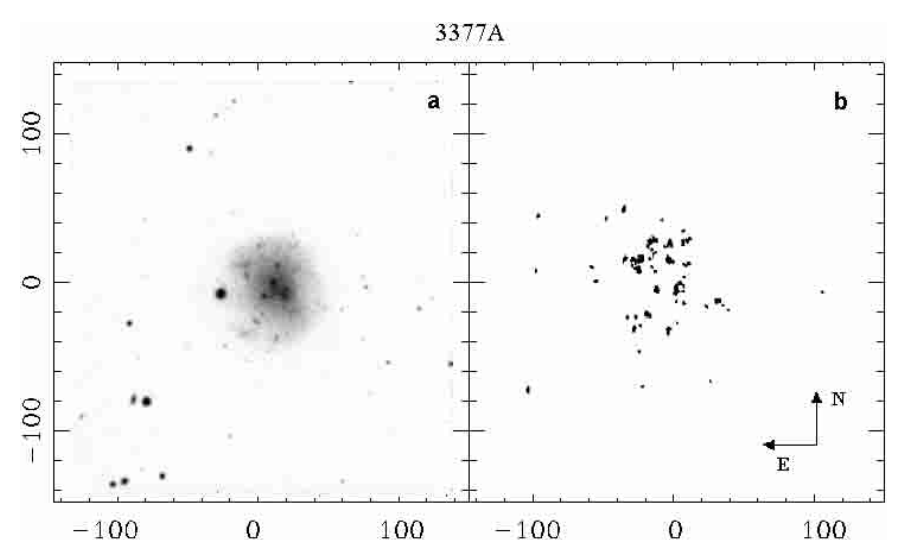

Fig. 1. Panel a) $B$ band image of NGC 3377A. Panel b) map of the identified YSGs. North is up and East to the left. Coordinates are in arcseconds and the offset is relative to the galactic centre.

The application of our algorithm to this galaxy led us to the identification of 83 YSGs, whose positions in the galaxy are shown in Fig. 1 and whose main characteristic parameters are given in Table 2 . We remind the reader that, in order to reduce the contamination by random groups in this list of YSGs candidates, we adopted (as in Paper I) the procedure described by Battinelli \& Demers (1992). This procedure results in the introduction of a threshold, $N_{\text {lim }}$, in the number of pixels in each groups, such that the contamination by random groups in the sample of all the groups composed of at least $N_{\text {lim }}$ pixels is less than $10 \%$. The computed $N_{\text {lim }}$ are $9,4,7$ for NGC 3377A, NGC 3507 and NGC 4394, respectively. Blue magnitudes and surface brightnesses have been corrected for a total extinction of $0.06 \mathrm{mag}$ (0.04 mag galactic extinction and $0.02 \mathrm{mag}$ internal extinction) as given in RC3. As explained in Paper I (Sect. 3), a precise determination of the integrated colours of YSGs is not easy since they often lie in areas with a lot of "structure".

We define the size, $D_{x y}$, of an YSG, as the average of the $x$ and $y$ extents; the size distribution is shown in Fig. 4 for the three galaxies studied in this paper. For NGC 3377A the average size is $87 \mathrm{pc}$ with a standard deviation of $59 \mathrm{pc}$. Fitting the high-tail of the size distribution with the power law $\mathrm{d} N \propto D_{x y}^{\alpha} \mathrm{d} D_{x y}$ we find $\alpha=-2.3 \pm 0.3$ (correlation coefficient $r=0.93$ ). The shaded strips shown in Fig. 4 are the $D_{x y}$ intervals directly affected by the introduction of the $N_{\text {lim }}$ threshold for each galaxy. Such shaded areas are therefore certainly incomplete.

Knezek et al. (1999) give a multi-color map of NGC 3377A where several H $\alpha$ "knots" are evident over the whole optical disk. A comparison of our YSG map with the $\mathrm{H} \alpha$ knots shows that a large percentage of the latter (about 80\%) correspond to YSGs. Two out of the six $\mathrm{H} \alpha$ sources that have no counterpart in our YSG sample are very close to a bright field star, three are in the very outskirts of the optical galaxy and just one lies along a spiral branch. This is a very tiny $\mathrm{H} \alpha$ region perhaps related to a YSG too small to be resolved by us. The most significant difference between our YSGs and Knezek 's sources

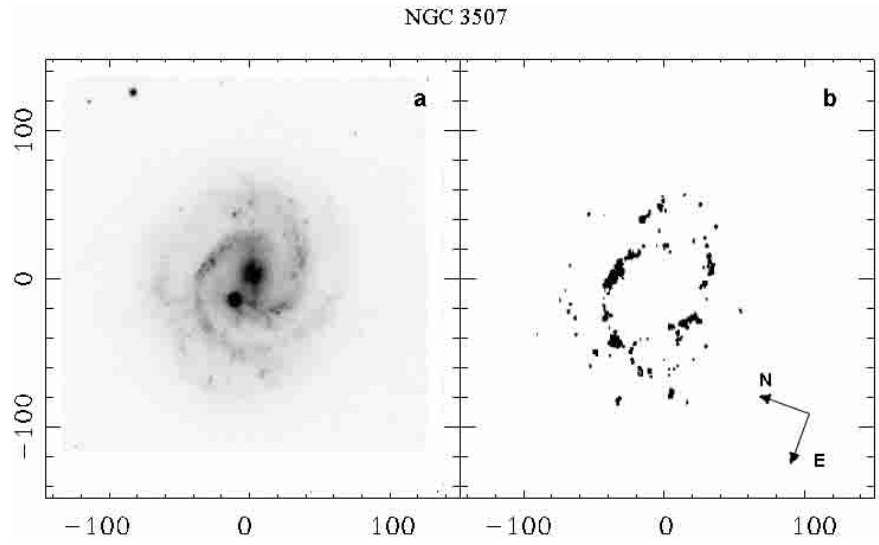

Fig. 2. Panel a) B band image of NGC 3507. Panel b) map of the identified YSGs. Orientation of the image is shown. The angle between the $y$-axis and North is $70^{\circ}$. Coordinates are in arcseconds and the offset is relative to the galactic centre.

is in two big clumps near the center that do not show well localized $\mathrm{H} \alpha$ emission, but are well characterized in our work.

\subsection{NGC 3507}

The spiral NGC 3507 is a typical barred galaxy (SBb type), with two long regular symmetric arms circling around the nucleus and the bar. The inner parts of each arm are more regular than the outer parts. The isophotal major axis is $D_{25}=3.4^{\prime}$ (RC3), the inclination is $26^{\circ}$ (Schulman et al. 1997) while the position angle is about $90^{\circ}$ (de Jong \& van der Kruit 1994). According to Garcia et al. (1993), NGC 3507 is the brightest member of a small group of galaxies. From the radial velocity of $906 \mathrm{~km} \mathrm{~s}^{-1}$ (RC3) and $H_{0}=75 \mathrm{~km} \mathrm{~s}^{-1} / \mathrm{Mpc}$, a distance of $12.1 \mathrm{Mpc}$ is obtained that corresponds to a scale of $16.4 \mathrm{pc} / \mathrm{pixel}$. Schulman et al. (1997) studied the HI distribution and dynamics in a small sample of galaxies, including NGC 3507. This paper concludes that in NGC 3507 there are no High Velocity Clouds (HVCs, i.e. HI regions with peculiar motion). Since HVCs are thought to be star formation indicators (probably originating in Supernova explosions) the lack of HVCs in NGC 3507 suggests a low star formation rate in this galaxy. Figure 2 shows the map of the 90 YSGs detected: most of the objects are located along the inner part of the arms. In Table 3 we give the main properties of the YSGs. Galactic and internal B-absorptions are taken to be $0.00 \mathrm{mag}$ and $0.10 \mathrm{mag}$, respectively, from RC3. The YSGs average size is $121 \mathrm{pc}$, with a standard deviation of $147 \mathrm{pc}$ and the slope of the fitted power law to the size distribution is the smallest one with $\alpha=-1.6 \pm 0.3$ $(r=0.80)$.

\subsection{NGC 4394}

NGC 4394 is a barred and ringed spiral galaxy, with integrated $B$ magnitude $11.53 \mathrm{mag}$ and with $D_{25}=3.6^{\prime}$ (RC3). Its proximity to M 85, just $6^{\prime}$ East, and their 
Table 2. YSGs of NGC 3377A. $N$ is the identification number, $X$ and $Y$ are the coordinates in arcsecs. $R$ is the galactocentric distance (in kpc), corrected for the inclination of the galaxy. $B$ is the total blue magnitudes. $\Sigma_{B}$ is the surface brightness (in mag/ $\operatorname{arcsec}^{2}$ ). Magnitudes are corrected by the extinction, from RC3. $D_{x y}$ is the linear dimension (in pc).

\begin{tabular}{|c|c|c|c|c|c|c|c|c|c|c|c|c|c|}
\hline$N$ & $X$ & $Y$ & $R$ & $B$ & $\Sigma_{B}$ & $D_{x y}$ & $N$ & $X$ & $Y$ & $R$ & $B$ & $\Sigma_{B}$ & $D_{x y}$ \\
\hline 1 & 39 & -127 & 6.9 & 24.56 & 24.29 & 72 & 43 & -26 & 12 & 1.51 & 23.65 & 24.14 & 86 \\
\hline 2 & 99 & -121 & 8.13 & 24.67 & 24.3 & 43 & 44 & 11 & 11 & 0.84 & 23.66 & 24.09 & 86 \\
\hline 3 & -130 & -96 & 8.42 & 24.4 & 24.33 & 72 & 45 & -163 & 12 & 8.49 & 23.22 & 23.15 & 57 \\
\hline 4 & -136 & -83 & 8.27 & 24.43 & 24.37 & 72 & 46 & 9 & 12 & 0.83 & 24.19 & 24.21 & 72 \\
\hline 5 & -166 & -82 & 9.61 & 24.56 & 24.5 & 72 & 47 & 12 & 13 & 0.97 & 23.44 & 24.37 & 115 \\
\hline 6 & -99 & -71 & 6.35 & 22.07 & 23.55 & 159 & 48 & -21 & 15 & 1.37 & 21.08 & 23.59 & 275 \\
\hline 7 & -19 & -68 & 3.71 & 24.64 & 24.58 & 57 & 49 & -32 & 14 & 1.86 & 24.36 & 24.1 & 72 \\
\hline 8 & 63 & -66 & 4.76 & 24.77 & 24.39 & 57 & 50 & -1 & 15 & 0.83 & 21.13 & 23.63 & 231 \\
\hline 9 & 28 & -65 & 3.69 & 24.65 & 24.39 & 57 & 51 & 2 & 14 & 0.78 & 23.74 & 24.52 & 101 \\
\hline 10 & -122 & -49 & 6.84 & 24.78 & 24.52 & 72 & 52 & -2 & 14 & 0.78 & 24.91 & 24.53 & 57 \\
\hline 11 & -21 & -45 & 2.62 & 23.21 & 23.64 & 86 & 53 & -26 & 15 & 1.59 & 21.84 & 23.56 & 173 \\
\hline 12 & -1 & -31 & 1.63 & 21.43 & 23.63 & 231 & 54 & -31 & 16 & 1.85 & 22.57 & 24.03 & 144 \\
\hline 13 & -25 & -31 & 2.1 & 24.58 & 24.6 & 72 & 55 & -14 & 16 & 1.15 & 23.97 & 24.29 & 86 \\
\hline 14 & -24 & -29 & 2 & 23.46 & 23.84 & 86 & 56 & -25 & 17 & 1.58 & 24.47 & 24.31 & 72 \\
\hline 15 & -21 & -28 & 1.84 & 24.48 & 24.1 & 57 & 57 & -21 & 17 & 1.46 & 22.71 & 23.93 & 130 \\
\hline 16 & 4 & -26 & 1.39 & 24.28 & 24.22 & 72 & 58 & -19 & 18 & 1.4 & 24.1 & 24.03 & 72 \\
\hline 17 & -29 & -22 & 1.93 & 24.27 & 24.29 & 72 & 59 & -12 & 18 & 1.15 & 24.52 & 24.14 & 57 \\
\hline 18 & -24 & -22 & 1.72 & 24.04 & 24.21 & 72 & 60 & -10 & 20 & 1.21 & 24.1 & 24.48 & 101 \\
\hline 19 & -15 & -20 & 1.35 & 21.38 & 23.83 & 231 & 61 & -12 & 22 & 1.34 & 23.57 & 24.21 & 101 \\
\hline 20 & 40 & -17 & 2.28 & 25.02 & 24.64 & 57 & 62 & -3 & 24 & 1.28 & 23.73 & 24.46 & 115 \\
\hline 21 & 25 & -15 & 1.53 & 23.71 & 24.2 & 86 & 63 & -15 & 24 & 1.52 & 22.86 & 24.25 & 130 \\
\hline 22 & 36 & -14 & 2.03 & 24.02 & 23.95 & 57 & 64 & 0 & 24 & 1.29 & 24.76 & 24.49 & 57 \\
\hline 23 & 9 & -13 & 0.84 & 23.08 & 23.72 & 101 & 65 & -1 & 25 & 1.34 & 24.13 & 24.31 & 72 \\
\hline 24 & 33 & -11 & 1.82 & 22.31 & 23.93 & 159 & 66 & 0 & 27 & 1.42 & 22.23 & 24.1 & 188 \\
\hline 25 & 4 & -11 & 0.65 & 24.18 & 23.91 & 57 & 67 & 8 & 25 & 1.4 & 24.6 & 24.53 & 72 \\
\hline 26 & -161 & -8 & 8.35 & 24.27 & 24.58 & 72 & 68 & -12 & 28 & 1.61 & 21.29 & 23.8 & 260 \\
\hline 27 & 3 & -4 & 0.31 & 21.41 & 24.08 & 289 & 69 & 8 & 27 & 1.47 & 23.95 & 24.05 & 72 \\
\hline 28 & -9 & -4 & 0.54 & 20.87 & 23.34 & 217 & 70 & 9 & 28 & 1.53 & 23.75 & 24.13 & 101 \\
\hline 29 & 106 & -5 & 5.5 & 23.86 & 23.79 & 57 & 71 & 12 & 29 & 1.64 & 22.5 & 24.07 & 188 \\
\hline 30 & 8 & -5 & 0.53 & 25.01 & 24.64 & 43 & 72 & -11 & 29 & 1.65 & 24.51 & 24.13 & 57 \\
\hline 31 & 9 & -1 & 0.49 & 25 & 24.62 & 57 & 73 & -12 & 31 & 1.73 & 24.62 & 24.24 & 57 \\
\hline 32 & 5 & 0 & 0.3 & 24.23 & 24.33 & 86 & 74 & 9 & 35 & 1.87 & 23.67 & 24.4 & 101 \\
\hline 33 & 7 & 0 & 0.39 & 24.44 & 24.37 & 72 & 75 & -44 & 43 & 3.24 & 22.96 & 23.89 & 115 \\
\hline 34 & 7 & 0 & 0.36 & 24.73 & 24.35 & 57 & 76 & -5 & 42 & 2.22 & 24.23 & 24.26 & 72 \\
\hline 35 & -52 & 1 & 2.7 & 22.51 & 24.04 & 144 & 77 & -92 & 45 & 5.33 & 22.27 & 23.52 & 115 \\
\hline 36 & 9 & 4 & 0.56 & 24.89 & 24.62 & 72 & 78 & -32 & 49 & 3.07 & 22.23 & 23.82 & 159 \\
\hline 37 & -22 & 8 & 1.25 & 21.1 & 23.61 & 246 & 79 & -118 & 94 & 7.85 & 24.88 & 24.61 & 57 \\
\hline 38 & -10 & 8 & 0.69 & 24.54 & 24.48 & 72 & 80 & -154 & 95 & 9.4 & 24.99 & 24.73 & 57 \\
\hline 39 & -94 & 8 & 4.89 & 24.22 & 24.15 & 57 & 81 & -149 & 115 & 9.8 & 24.13 & 24.44 & 101 \\
\hline 40 & -54 & 10 & 2.9 & 23.32 & 24.1 & 86 & 82 & 35 & 125 & 6.73 & 24.37 & 24.11 & 57 \\
\hline 41 & -12 & 10 & 0.87 & 24.29 & 24.66 & 86 & 83 & 77 & 130 & 7.85 & 24.21 & 24.05 & 57 \\
\hline 42 & -21 & 11 & 1.27 & 24.79 & 24.53 & 57 & & & & & & & \\
\hline
\end{tabular}


Table 3. YSGs of NGC 3507. See caption of Table 2.

\begin{tabular}{|c|c|c|c|c|c|c|c|c|c|c|c|c|c|}
\hline$N$ & $X$ & $Y$ & $R$ & $B$ & $\Sigma_{B}$ & $D_{x y}$ & $N$ & $X$ & $Y$ & $R$ & $B$ & $\Sigma_{B}$ & $D_{x y}$ \\
\hline 1 & -30 & -75 & 4.76 & 19.88 & 22.26 & 229 & 46 & -65 & -17 & 4.26 & 21.75 & 22.3 & 98 \\
\hline 2 & 17 & -76 & 4.77 & 22.67 & 22.69 & 81 & 47 & 54 & -17 & 3.73 & 22.18 & 22.67 & 81 \\
\hline 3 & 5 & -72 & 4.34 & 20.3 & 22.31 & 196 & 48 & -63 & -12 & 4.13 & 21.57 & 22.38 & 114 \\
\hline 4 & 6 & -69 & 4.19 & 20 & 22.19 & 212 & 49 & -71 & -10 & 4.6 & 22.8 & 22.53 & 65 \\
\hline 5 & -7 & -59 & 3.56 & 21.88 & 22.37 & 98 & 50 & 30 & -10 & 2.11 & 23.5 & 22.48 & 32 \\
\hline 6 & -14 & -58 & 3.51 & 21.03 & 22.33 & 147 & 51 & -40 & -9 & 2.64 & 23.58 & 22.32 & 32 \\
\hline 7 & 4 & -58 & 3.52 & 22.7 & 22.53 & 65 & 52 & -37 & -5 & 2.44 & 23.4 & 22.38 & 32 \\
\hline 8 & -16 & -58 & 3.54 & 22.77 & 22.61 & 81 & 53 & -36 & -5 & 2.38 & 23.52 & 22.5 & 48 \\
\hline 9 & -8 & -56 & 3.35 & 20.53 & 22.23 & 163 & 54 & -67 & -4 & 4.34 & 24.02 & 22.77 & 32 \\
\hline 10 & -15 & -54 & 3.35 & 20.81 & 22.34 & 163 & 55 & -33 & 5 & 2.2 & 16.82 & 21.92 & 1032 \\
\hline 11 & 8 & -55 & 3.36 & 21.74 & 22.33 & 81 & 56 & 28 & 0 & 1.86 & 19.69 & 22.22 & 294 \\
\hline 12 & 4 & -55 & 3.31 & 23.8 & 22.54 & 32 & 57 & -38 & -2 & 2.45 & 23.49 & 22.24 & 32 \\
\hline 13 & -49 & -53 & 4.36 & 21.84 & 22.48 & 98 & 58 & -27 & 3 & 1.77 & 23 & 22.49 & 48 \\
\hline 14 & 10 & -52 & 3.26 & 22.54 & 22.47 & 65 & 59 & 34 & 9 & 2.25 & 18.94 & 22 & 376 \\
\hline 15 & -20 & -49 & 3.14 & 21.29 & 22.38 & 163 & 60 & 28 & 10 & 1.92 & 21.95 & 22.12 & 81 \\
\hline 16 & 1 & -49 & 2.95 & 23.97 & 22.95 & 48 & 61 & 32 & 11 & 2.13 & 21.62 & 22.26 & 98 \\
\hline 17 & 21 & -49 & 3.31 & 23.18 & 22.67 & 48 & 62 & -63 & 11 & 4.22 & 22.87 & 22.71 & 65 \\
\hline 18 & -36 & -46 & 3.48 & 23.31 & 22.8 & 65 & 63 & -27 & 13 & 1.97 & 22.56 & 22.18 & 65 \\
\hline 19 & -46 & -44 & 3.84 & 20.28 & 22.4 & 212 & 64 & 33 & 14 & 2.24 & 20.01 & 22.11 & 196 \\
\hline 20 & -21 & -43 & 2.83 & 20.56 & 22.33 & 180 & 65 & -25 & 15 & 1.97 & 22.9 & 22.39 & 48 \\
\hline 21 & -32 & -36 & 2.94 & 17.75 & 22.1 & 622 & 66 & -20 & 18 & 1.77 & 18.38 & 22.09 & 491 \\
\hline 22 & -26 & -40 & 2.86 & 21.39 & 22.31 & 98 & 67 & 32 & 18 & 2.29 & 20.39 & 22.16 & 180 \\
\hline 23 & 30 & -40 & 3.2 & 21.68 & 22.37 & 98 & 68 & 33 & 17 & 2.35 & 22.47 & 22.31 & 81 \\
\hline 24 & -19 & -38 & 2.55 & 21.21 & 22.43 & 130 & 69 & 5 & 20 & 1.24 & 23.13 & 22.47 & 48 \\
\hline 25 & 10 & -39 & 2.48 & 22.46 & 22.56 & 81 & 70 & 1 & 22 & 1.33 & 21.81 & 22.18 & 81 \\
\hline 26 & 11 & -36 & 2.33 & 20.29 & 22.22 & 212 & 71 & -13 & 24 & 1.73 & 21.28 & 22.17 & 98 \\
\hline 27 & -14 & -36 & 2.3 & 23.81 & 22.79 & 48 & 72 & 3 & 24 & 1.46 & 20.19 & 22.11 & 163 \\
\hline 28 & -10 & -36 & 2.2 & 23.51 & 22.7 & 32 & 73 & 31 & 24 & 2.41 & 20.38 & 21.97 & 147 \\
\hline 29 & 13 & -34 & 2.27 & 22.44 & 22.61 & 81 & 74 & 0 & 24 & 1.48 & 22.48 & 22.32 & 65 \\
\hline 30 & 10 & -31 & 2.05 & 20.44 & 22.18 & 163 & 75 & -3 & 25 & 1.53 & 22.73 & 22.07 & 48 \\
\hline 31 & -59 & -32 & 4.19 & 21.95 & 22.59 & 98 & 76 & 22 & 27 & 2.1 & 22.03 & 22.4 & 81 \\
\hline 32 & -86 & -32 & 5.78 & 24.33 & 23.08 & 32 & 77 & 29 & 29 & 2.51 & 21.68 & 22.32 & 114 \\
\hline 33 & 6 & -29 & 1.83 & 23.01 & 22.19 & 48 & 78 & -1 & 34 & 2.07 & 23.32 & 22.81 & 48 \\
\hline 34 & 6 & -27 & 1.72 & 19.53 & 22 & 262 & 79 & 37 & 36 & 3.14 & 20.58 & 22.17 & 147 \\
\hline 35 & -36 & -28 & 2.81 & 23.2 & 22.38 & 48 & 80 & -13 & 42 & 2.71 & 18.85 & 21.76 & 278 \\
\hline 36 & 15 & -26 & 1.91 & 18.52 & 21.68 & 393 & 81 & -9 & 44 & 2.78 & 20.18 & 22.11 & 196 \\
\hline 37 & 20 & -22 & 1.95 & 18.7 & 21.95 & 376 & 82 & -50 & 45 & 4.39 & 21.18 & 22.33 & 114 \\
\hline 38 & 25 & -24 & 2.26 & 20.02 & 22.17 & 212 & 83 & -39 & 45 & 3.82 & 23.32 & 22.5 & 32 \\
\hline 39 & 14 & -24 & 1.78 & 23.11 & 22.09 & 48 & 84 & 2 & 47 & 2.8 & 22.34 & 22.36 & 81 \\
\hline 40 & -39 & -24 & 2.82 & 23.34 & 22.32 & 32 & 85 & -1 & 49 & 2.97 & 19.51 & 22.18 & 262 \\
\hline 41 & -39 & -19 & 2.73 & 18.97 & 22.18 & 475 & 86 & 26 & 51 & 3.38 & 20.52 & 22.18 & 147 \\
\hline 42 & -60 & -22 & 4.03 & 21.24 & 22.2 & 98 & 87 & 0 & 54 & 3.21 & 21.25 & 22.31 & 130 \\
\hline 43 & -36 & -20 & 2.56 & 23.06 & 22.41 & 48 & 88 & 0 & 56 & 3.37 & 23.34 & 22.52 & 48 \\
\hline 44 & -35 & -19 & 2.49 & 22.26 & 22.43 & 81 & 89 & 15 & 57 & 3.5 & 23.37 & 22.55 & 48 \\
\hline 45 & 22 & -19 & 1.93 & 22.01 & 22.25 & 81 & 90 & 16 & 58 & 3.56 & 24.14 & 22.88 & 32 \\
\hline
\end{tabular}




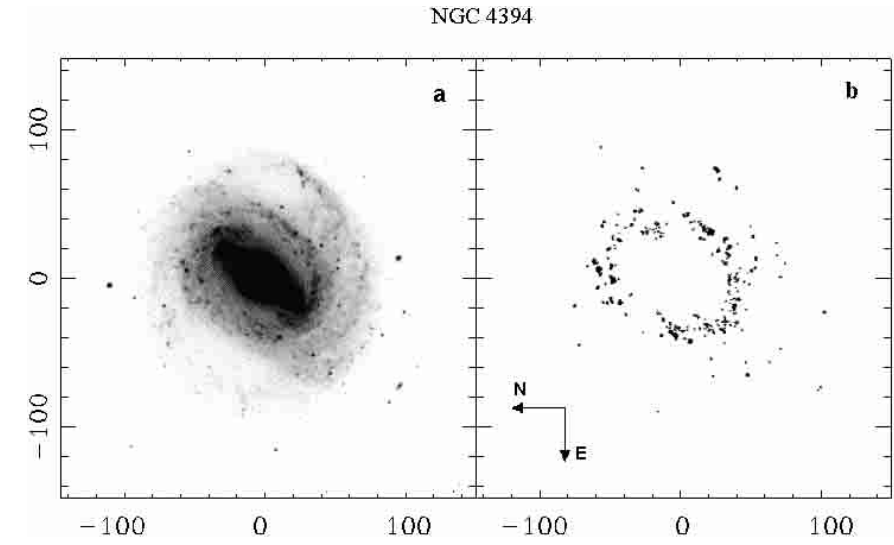

Fig. 3. Panel a) $B$ band image of NGC 4394. Panel b) map of the identified YSGs. North is left and East is down. Coordinates are in arcseconds and the offset is relative to the galactic centre.

similar radial velocities suggest that NGC 4394 may belong to the M 85 subgroup of the Virgo Cluster. Thus we assume as the distance of this galaxy that deduced by Ferrarese et al. (2000) (about $16 \mathrm{Mpc}$ ) for the M 85 subcluster based on Cepheid indicators. The inclination and the position angle are taken from Chapelon et al. (1999) and are $25^{\circ}$ and $103^{\circ}$, respectively.

The distribution of the YSGs, shown in Fig. 3, is clearly concentrated in the ring region and just few YSGs are detected along the spiral arms. This is supported by the radial distribution of the surface luminosity density, $\sigma_{B}$, defined as the total $B$ luminosity of the YSGs found in an annulus divided by its area (see Fig. 5). This quantity is clearly related to the high-mass star formation rate. The concentrated distribution of the YSGs probably reflects the high abundance of cold gas detected in the rings of galaxies (Wong et al. 2000). Moreover, HII regions were studied by Hodge (1974) in NGC 4394 with a $2.1 \mathrm{~m}$ telescope. He also found a clear concentration of the HII regions in the ring, few of them being distributed in the outer arms.

Table 4 gives the YSG parameters; the average YSG diameter is $114 \mathrm{pc}$ with a standard deviation of $77 \mathrm{pc}$, while the exponent $\alpha$ of the power law is $-2.7 \pm 0.3(r=$ $0.92)$.

\section{Discussion and conclusions}

With our semi-automatic identification algorithm we have determined the population and main characteristics of Young Star Groupings in the spiral galaxies NGC 3377A, NGC 3507 and NGC 4394. The data collected constitute not only an enlargement of the YSG data-base but also a direct source of astrophysical information about the properties of the regions of star formation in spirals.

The analysis of the YSG differential $B$ luminosity functions (Fig. 6) shows that their high luminosity tails can

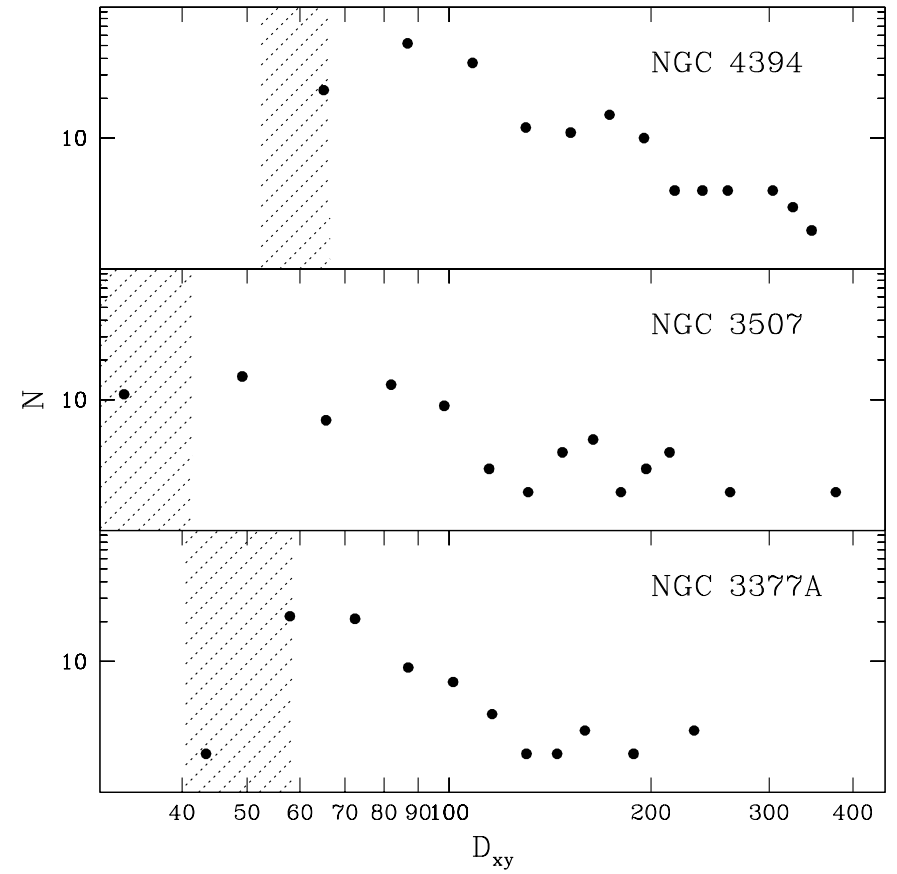

Fig. 4. Size distribution of the YSGs. The size (in pc) is defined as the average of the $x$ and $y$ extents of the YSG. Shaded areas are the incompleteness strips as described in the text.

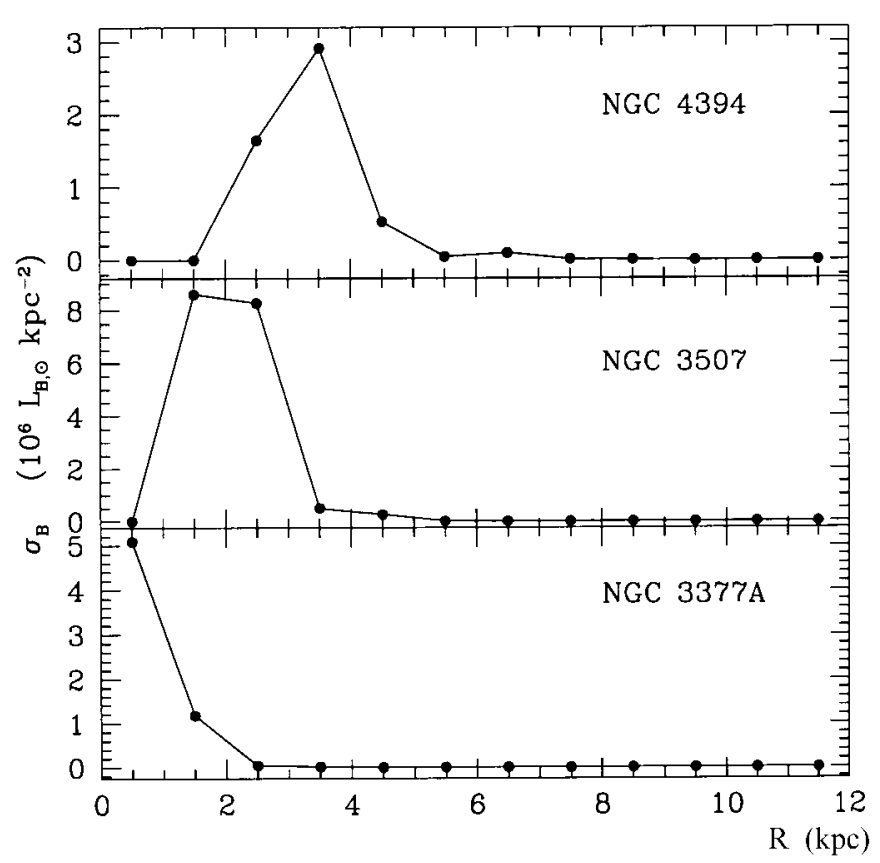

Fig. 5. The surface luminosity density $\left(\sigma_{B}\right.$, in $\left.10^{6} L_{B, \odot} \mathrm{kpc}^{-2}\right)$ of YSGs averaged over concentric circular annuli as a function of the galactrocentric radius ( $R$, in $\mathrm{kpc}$ ) in NGC 3377A, NGC 3507 and NGC 4394. The YSG positions were corrected for the galaxy inclination.

be well fitted with power laws $\mathrm{d} N \propto L_{B}^{\beta} \mathrm{d} L_{B}$. We find $\beta=-1.79 \pm 0.10,-1.31 \pm 0.08$ and $-1.90 \pm 0.18$, for NGC 3377A, NGC 3507 and NGC 4394, respectively (the correlation coefficients $r$ are equal to 0.94, 0.99 and 0.99). These values are in good agreement with those found by 
Table 4. YSGs of NGC 4394. See caption of Table 2.

\begin{tabular}{|c|c|c|c|c|c|c|c|c|c|c|c|c|c|c|c|c|c|c|c|c|}
\hline & $X$ & $Y$ & $R$ & $B$ & $\Sigma_{B}$ & $D_{x y}$ & & $X$ & $Y$ & $R$ & & $\Sigma_{B}$ & $D_{x y}$ & $N$ & $X$ & & & & & $\bar{x}_{x}$ \\
\hline & 50 & -87 & 7.91 & 21.97 & 22.51 & & 3 & -35 & & 3.42 & & & & 125 & -33 & & 3.54 & & & \\
\hline 2 & 28 & & & & & & & & & & & & & & & & & & & \\
\hline 3 & 21 & & & 59 & .93 & & & 36 & & & & 34 & & & & & & & & \\
\hline 4 & 30 & 66 & & .14 & 22.78 & 30 & & 25 & -14 & 49 & 3.53 & 3.15 & 77 & 28 & -20 & & 86 & 2.48 & 2.32 & \\
\hline 5 & 42 & 60 & 02 & .5 & 9 & 73 & & 69 & -14 & 06 & & 3.23 & & 29 & -29 & 0 & 38 & 2.55 & 2.65 & \\
\hline 6 & 26 & -59 & 4 & .6 & .22 & 37 & & -55 & -11 & & .47 & 2.93 & 173 & 30 & -36 & 31 & & & & \\
\hline 7 & 24 & -58 & 92 & .92 & 3.09 & & & 45 & -11 & 3.91 & 2.64 & & 08 & 31 & -42 & & & & & \\
\hline 8 & 0 & -50 & 92 & .87 & 2.84 & 52 & & -35 & -11 & 3.2 & 3.47 & 22.96 & 55 & 32 & -41 & & 4.28 & 2.37 & 3.06 & 7. \\
\hline & 22 & -44 & .9 & 0.99 & 22.77 & 39 & & 54 & -9 & 4.63 & 0.62 & 22.75 & 325 & & 3 & & .56 & 2.53 & 2.8 & \\
\hline & 26 & -44 & 04 & 1.66 & 22.87 & 7 & & 32 & -10 & 2.93 & 3.2 & 23.04 & & & -20 & & 06 & 2.03 & & \\
\hline & & & 41 & & 2 & & & 34 & -8 & 03 & & .62 & & & -22 & & & & & \\
\hline & 6 & & 28 & .86 & 3 & & & & $-\varepsilon$ & 43 & & 23.0 & & & & & 07 & & 23 & \\
\hline & 38 & 42 & & 2 & 2 & 3 & & 42 & -6 & & & 22.74 & & & & & 86 & . & & \\
\hline & & & 74 & 5 & & & & 10 & -7 & & & 22.77 & & & -29 & & & & & \\
\hline & & & & .29 & & 87 & & 59 & -6 & & & 3.15 & & & 2 & & 66 & 0.15 & & \\
\hline & & 37 & 34 & .09 & .83 & 87 & & -54 & -5 & & 3 & 22.27 & 130 & & -24 & & .29 & 2.8 & 2.42 & 37 \\
\hline & 7 & 36 & & 24 & .01 & 130 & & 51 & -4 & & 55 & 22.32 & 47 & 11 & -1 & & .75 & 1.59 & 22.75 & 17 \\
\hline & & & 16 & 31 & .05 & 87 & 8 & -44 & -5 & 3.83 & 3.07 & 23 & & 12 & -25 & & & 3.04 & 2.6 & 37 \\
\hline & & 36 & 25 & 3.55 & 3.05 & 87 & & 43 & -4 & 3.7 & 3.09 & 22.83 & 08 & & 5 & & 7 & 3.52 & 37 & 55 \\
\hline & & 36 & 04 & 2.21 & 22.85 & 10 & & & -3 & 4.45 & 1.94 & 23.1 & 173 & 44 & -40 & & 33 & 3.12 & 66 & 87 \\
\hline & 12 & 35 & 93 & .13 & .57 & 95 & & & -1 & 23 & 32 & & & 15 & -8 & & & & & \\
\hline & 5 & -35 & 79 & 3.47 & 23.1 & 87 & & & -1 & & 1 & & & & & & & & & 5 \\
\hline & 17 & 35 & & 33 & 22.87 & 13 & & & 0 & 4.26 & & 22.98 & & & -17 & & .11 & 3.35 & 2.8 & 37 \\
\hline & 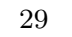 & 34 & & .48 & 2.8 & & & & & 3 & & 22.75 & & & -3 & & 8 & 22.2 & 2. & 3 \\
\hline & & & & 93 & . & & & 3 & & & & 22.72 & & & & & 8 & 3.37 & & 5 \\
\hline & & & & & . & & & & & & & 22.44 & & & & & $\varepsilon$ & .49 & & 5 \\
\hline & 20 & & & & & & & & & & & & & & -34 & & & & & 37 \\
\hline & 51 & 33 & & 6 & 3. & 0 & & 4 & & & & 22. & & & -20 & & & 24 & & 0 \\
\hline & 0 & 33 & 71 & & 22.72 & & & ; & & 3.23 & 3. & 22.98 & & & -31 & & & & & 95 \\
\hline & 24 & 29 & & 2 & & & & 4 & & & & 22.73 & & & 0 & & & & & 55 \\
\hline & 9 & 32 & & & 6 & & & 3 & & & & 1 & & & 17 & & & & & 37 \\
\hline & 33 & 33 & 73 & & 3 & & & & & & & 1 & & & -13 & & & & & 5 \\
\hline & & -32 & 7 & .15 & 2.5 & 65 & & 3 & & & & & 173 & 77 & -45 & & & & & \\
\hline & & 31 & 74 & .27 & & & & 46 & & & & & & & -24 & & & & & $0 \varepsilon_{-}$ \\
\hline & & -31 & & & & 8 & & 50 & & & & & & & -8 & & & & & 0 \\
\hline & & -31 & 69 & 3 & 2. & & & 52 & & & & & & & -23 & & 4 & & & 0 \\
\hline & & -30 & & 6 & 22. & & & & & & & 2.8 & & & - & & & & & 37 \\
\hline & & -30 & 95 & .67 & 2. & 5 & & & & & & & & & -6 & & & 2.6 & & 3 \\
\hline & & -30 & & .96 & & 8 & & 4 & & & & & & & & & & 23.71 & & 37 \\
\hline & 1 & -29 & & .25 & 2 & & & & & & & & & & & & & & & \\
\hline & 9 & -29 & & & & & & 3 & & & & & & & -33 & & & & & 5 \\
\hline & & 28 & & & & & & 30 & & & & & & & . & & & & & \\
\hline & & 28 & & & & & & & & & & & & & -5 & & & & & 0 \\
\hline & 7 & -29 & & .44 & & & & 4. & & & & & & & 21 & & & & & 87 \\
\hline & & -29 & & & & & & 3 & & & & & & & & & & & & $7:$ \\
\hline & & 27 & & .83 & & & & & & & & & & & -20 & & & & & 37 \\
\hline & 53 & -26 & 06 & & & & & -3 & & & & & & & -7 & & & & & 10 \\
\hline & & -26 & & & & & & & & & & & & & -1 & & & & & \\
\hline & & 25 & 53 & & 22 & & & & & & & & & & 0 & & & & & 7 \\
\hline & & 25 & 62 & .78 & & 23 & & & & & & & & & -10 & & & & & 60 \\
\hline & & & & & & & & -4 & & & & & & & -33 & & & & & 55 \\
\hline & & & & & & & & & & & & & & & 64 & & & & & \\
\hline & & & & & & & & & & & & & & & -71 & & & & & 3 \\
\hline & & -25 & & & & & & & & & & & & & -24 & & & & & \\
\hline & & 23 & & & & & & & & & & & & & & & & & & \\
\hline & & 22 & & & & & & & & & & & & & -48 & & & & & \\
\hline & 42 & 21 & & & & 87 & & -10 & & & & & & & -49 & & & & & 9 \\
\hline & 37 & -19 & & & & 26 & & 12 & & & & & & & -26 & & & & & $0 \varepsilon$ \\
\hline & & -20 & & & & & & -4 & & & & & & & - & & & & & 0 \\
\hline & & - & & & & & & 1 & & & & & & & -97 & & & & & \\
\hline & \pm 0 & -1 & & & & 87 & & & & & & & & 185 & 11 & 93 & 7.42 & $3.5:$ & 23.17 & 55 \\
\hline & -29 & & 2.91 & 21.01 & 22.71 & & & 15 & & 2.48 & & 2.2 & & & & & & & & \\
\hline
\end{tabular}




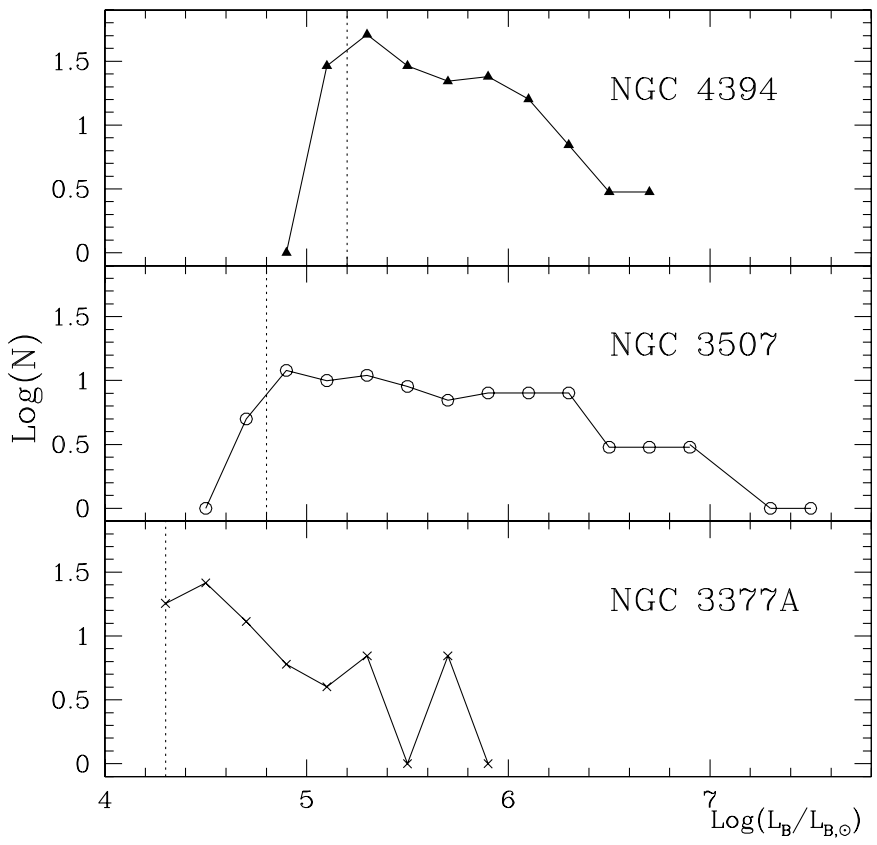

Fig. 6. The differential luminosity function of the YSGs in $B$-band. Crosses, circles and triangles refer to NGC 3377A, NGC 3507 and NGC 4394, respectively.

Elmegreen \& Salzer (1999) for the star forming complexes in a sample of 11 galaxies. Again, the introduction of the $N_{\text {lim }}$ threshold described in Sect. 3.1 implies a corresponding detection limit in the YSG luminosity. We evaluated such detection limits for each galaxy by plotting the luminosity as a function of number of pixels of the YSGs and then determining the value of the luminosity corresponding to $N_{\text {lim }}$. The detection limits, the dotted lines in Fig. 6, are close to the peaks of the luminosity functions, implying statistical incompleteness in those regions.

The sample of six spirals studied so far (three in Paper I and three in this paper) allows us a preliminary investigation of the existence of correlations among the YSG and parent galaxy properties. In Fig. 7 we show some evidence of positive correlations among the number, dimension, integrated $B$ magnitude and the total area of the YSG populations with global characteristics of the galaxies (integrated $B$ magnitude and dimension).

Incidentally, we note that in Fig. 7 the two ringed galaxies, NGC 7217 and NGC 4394, are close to each other and, at least in panels b and d, they seem to behave differently from the rest of the sample. Of course, a larger number of ringed galaxies is necessary to check how real such a behaviour is. In particular, the presence of these two galaxies in our small sample results in a shallower slope of the correlation between the size of the largest YSG in a galaxy and the galaxy $M_{B}$ with respect to the one obtained by Elmegreen et al. (1994). They claimed that their correlation closely matches the expected variation in the characteristic length of the gaseous gravitational instability with
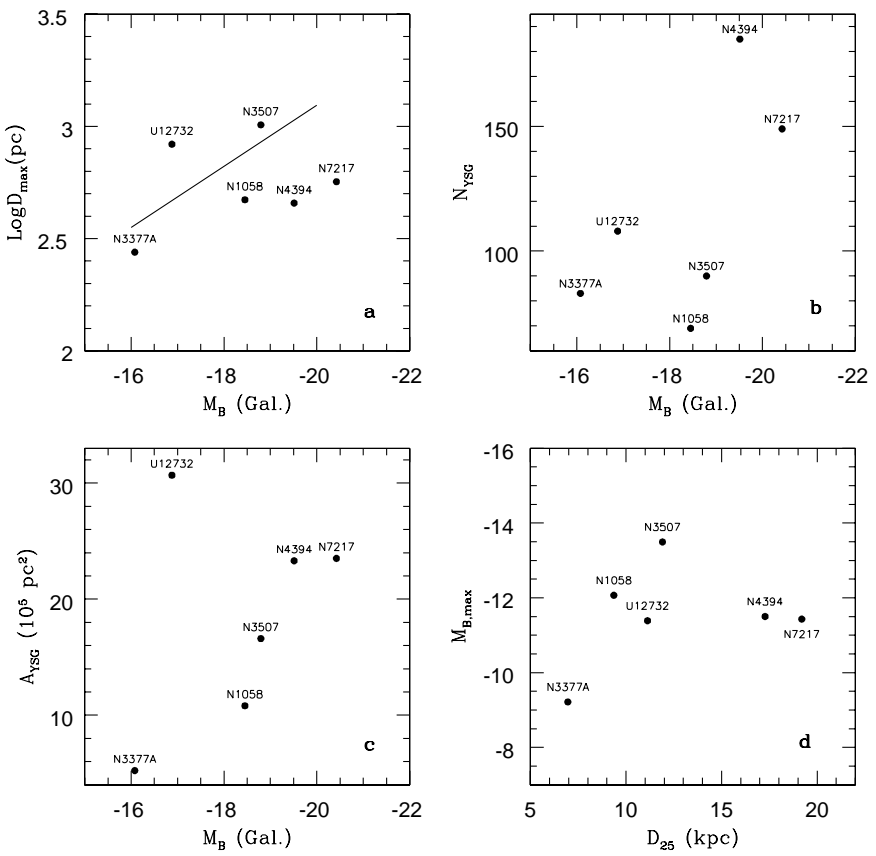

Fig. 7. Correlations between YSG and parent galaxy properties. Panel a) Logarithm of the size of the largest YSG vs. the integrated $B$ magnitude of the galaxy $\left(M_{B}\right)$; the straight line is the Elmegreen et al. (1994) relation (see text). Panel b) Number of YSGs $\left(N_{\mathrm{YSG}}\right)$ vs. $M_{B}$. Panel c) Total area of the YSG $\left(A_{\mathrm{YSG}}\right)$ vs. $M_{B}$. Panel d) Blue magnitude of the brightest YSG $\left(M_{B, \max }\right)$ vs. the diameter of the galaxy $\left(D_{25}\right)$ taken from RC3.

$M_{B}$. Such a correlation has been suggested by Selman \& Melnick (2000) to be the result of a size-of-sample effect acting on a universal size distribution $\left(\mathrm{d} N \propto D_{x y}^{-4.2} \mathrm{~d} D_{x y}\right)$. In order to check this suggestion, we can compare the -4.2 slope with those of the fitted power laws to the size distributions of the YSGs in NGC 3377A, NGC 3507 and NGC 4394, limited to the statistically reliable size ranges. The slopes found $(-2.3,-1.6,-2.7$ for NGC 3377A, NGC 3507, and NGC 4394, respectively) are quite close to the value, -2 , for a star forming molecular cloud size distribution (see Solomon et al. 1987; Larson 1981). They are always too shallow to explain the Elmegreen et al. (1994) relation by means of the size-of-sample effect. We stress, however, that our six points in Fig. 7a suggest a slope not as steep as Elmegreen et al.'s relation. However, we remark that our sample is still too small to draw firm conclusions about this question.

\section{References}

Adanti, S., Battinelli, P., Capuzzo-Dolcetta, R., \& Hodge, P. W. 1994, A\&AS, 108, 395

Battinelli, P., Capuzzo-Dolcetta, R., Hodge, P. W., Vicari, A., \& Wyder, T. K. 2000, A\&A, 357, 437

Battinelli, P., \& Demers, S. 1992, AJ 104, 1458

Chapelon, S., Contini, T., \& Davoust, E. 1999, A\&A, 345, 81 
de Jong, R., \& van der Kruit, P. C. 1994, AAS, 101, 451

de Vaucouleurs, G., de Vaucouleurs, A., Corwin, H. G. Jr., et al. 1991, Third Reference Catalogue of Bright Galaxies (Berlin: Springer-Verlag)

Elmegreen, D. M., Elmegreen, B. G., Lang, C., \& Stephens, C. 1994, ApJ, 425, 57

Elmegreen, B. G., Elmegreen, D. M., Salzer, J. J., \& Mann, H. 1996, ApJ, 467, 579

Elmegreen, D. M., \& Salzer, J. J. 1999, AJ, 117, 764

Ferrarese, L., Ford, C. H., Huchra, J., Kennicutt, R. C. Jr., Mould, J. R., et al. 2000, ApJS, 128, 431

Garcia, A. M. 1993, A\&AS, 100, 47

Hodge, P. W. 1986, in Luminous Stars and Associations in Galaxies, IAU Symp. 116 (Dordrecht: Reidel)

Hodge, P. W. 1974, ApJS, 27, 113
Knezek, P. M., Sembach, K. R., \& Gallagher, J. S. 1999, A\&AS, 514,119

Landolt, A. U. 1992, AJ, 104, 340

Larson, R. B. 1981, MNRAS, 257, 119

Sandage, A., \& Hoffman, G. L. 1991, ApJ, 379, L45

Schulman, E., Bregman, J. N., \& Roberts, M. S. 1994, ApJ, 423,180

Selman, F. J., \& Melnick, J. 2000, ApJ, 534, 703

Solomon, P. M., Rivolo, A. R., Barret, J., \& Yahil, A. 1987, ApJ, 319, 730

Tonry, J. L., Blakeslee, J. P., Ajhae, E. A., \& Dressler, A. 1997, ApJ, 475, 399

Young, J. S., Allen, L., Jeffrey, D. P., Lesser, A., \& Rownd, B. 1996, AJ, 112, 1903

Wong, T., \& Blitz, L. 2000, ApJ, 540, 771 